\title{
A Finite State Space Model for Representing the Broadband Infinite Series for Acoustic Reverberation between Parallel Reflecting Planes
}

\author{
Michael J. Panza (D) \\ Gannon University, 109 University Square, Erie, PA 16541, USA \\ Correspondence should be addressed to Michael J. Panza; panza@gannon.edu
}

Received 27 November 2017; Revised 31 March 2018; Accepted 6 May 2018; Published 4 June 2018

Academic Editor: Salvatore Alfonzetti

Copyright (C) 2018 Michael J. Panza. This is an open access article distributed under the Creative Commons Attribution License, which permits unrestricted use, distribution, and reproduction in any medium, provided the original work is properly cited.

\begin{abstract}
The acoustic reverberation between two parallel reflecting planes can be represented by an infinite series of the images caused by the planes. To provide a more useful model for analysis and control, the infinite series version of the Green's function is converted into a finite state space model that retains the high frequency character that enables broadband noise inputs to be examined. The infinite series is first summed into a very accurate, approximate closed form expression in the time domain in terms of a radical function. The radical is then transformed into an expression containing exponentials which have exact Laplace transforms that lead to an overall closed form transfer function for the system. The system transfer function is transformed into a third-order state space model that theoretically contains all of the frequency characteristics of the infinite series representation. The accuracy of the state space model is examined by comparing it to the infinite series solution for three typical types of acoustical inputs: exponential for impulse noise, single frequency sine for harmonic noise, and a multifrequency Schroeder phased harmonic sequence for random noise.
\end{abstract}

\section{Introduction}

Finite state space models for systems have several important advantages. These include both single-input single-output and multi-input multi-output systems which can be formally treated. It is best suited both for the theoretical treatment of control systems (optimization, output feedback, and estimation with observers) and for numerical simulations. The determination of the system initial condition response is straightforward. It gives a better insight into the inner system behavior such as controllability, observability, and system characteristics. The use of finite state space models to represent partially or fully bounded acoustic and/or structural vibration systems for investigating lower frequency behavior has previously received attention. Several noted examples for truncated state space models used for lower frequencies are included below. Five modes from second-order modal differential equations along a bounded string are used in pole placement output and its derivative for state space feedback control design via an observer [1]. A few secondorder modal differential equations for waves in a bounded one-dimensional acoustic duct are coupled with speaker dynamics in a model to investigate low frequency behavior [2-4]. In [4], output feedback control using $\mathrm{H}_{\infty}$ and lag compensation are examined. A discrete model of acoustic waves in a bounded one-dimensional duct using five sections of the duct and ten state equations is presented in [5]. Finite element modeling is used in [6] to develop over twenty-four thousand coupled second-order modal differential equations for a two-dimensional bounded structure coupled to a threedimensional bounded acoustic space. A state space model using 214 states is then used to investigate frequencies up to $400 \mathrm{~Hz}$. Finite element modeling is used in [7] to develop many coupled second-order modal differential equations for a one-dimensional bounded structure coupled to the several electromagnetic piezoelectric patches. A state space model using a finite number of states is then used to investigate the performance of optimal LQ controller frequencies for up to $300 \mathrm{~Hz}$. Most of the above studies are targeted for lower frequencies.

The use of the Euler-Maclaurin sum formula to provide an approximate, accurate closed form solution for the 
infinite series Green's function that describes partially or fully bounded acoustic and structural systems is developed and verified in [8-10] by comparing the closed form solution to the infinite series solution for several types of inputs. In [8], the main part and associated variations of the EulerMaclaurin closed form with the infinite series solutions in a partially bounded three-dimensional acoustic space between two parallel plates are presented for an exponential input. In [9], the Euler-Maclaurin closed form solution is compared with the infinite series solutions in a partially bounded two-dimensional space acoustic space for a narrow duct. The comparison includes both the time and frequency domains for an exponential input. It also includes a broad range (up to $1000 \mathrm{~Hz}$ ) transfer function based comparison of the Euler-Maclaurin closed form with the infinite series for feed forward control of a disturbance and closed loop output feedback control. In [10], Euler-Maclaurin closed form representations for the Green's functions in both onedimensional acoustic and structural systems are determined in Laplace transform domain in terms of time integrals. The Laplace transform integrals are solved numerically and used in a broad range (up to $1000 \mathrm{~Hz}$.) transfer function based feed forward control system to meet a desired output for an input disturbance.

For all of the Euler-Maclaurin based studies in [8-10], state space representations were not considered. In view of the Euler-Maclaurin closed form results given in [8-10], in both time domain response and the transfer function based control for frequencies up to $1000 \mathrm{~Hz}$, and the successes in [1-7] for the truncated state space models for various types of low frequency control in acoustic and structural systems, the work in [11] focuses on the development of a low order but full finite state space model that would naturally include a broadband frequency range and essentially all of the terms required for the convergent infinite series in the Green's function representation of bounded structures. Also, in [11], a one-dimensional structural system of a string is used to develop a suitable state space for a two-dimensional plate. The developed state space model in [11] is shown to give an accurate trend for representing of the series transfer function for high frequencies up to $2000 \mathrm{~Hz}$. and the accurate time response for both a direct problem with an exponential input and for use in an observer based inverse problem of estimating a disturbance input.

In this paper, the ideas and approach in [11] for structural systems are expanded, modified, and improved to develop a low order but full finite space model that would naturally include a broadband frequency range and essentially of all the terms required for a convergent infinite series in the Green's function representation of the acoustic field between reflecting planes commonly known as reverberation. The distinguishing difference between this paper and the development in [11] is that, in [11], the Euler-Maclaurin sum formula is applied to an infinite series of string modes. In this paper, the Euler-Maclaurin sum formula is applied directly to the Laplace transform form of the Green's function infinite series for acoustic reverberation in [8] providing all polynomial type functions in the Laplace transform domain which are directly combined into a system function.

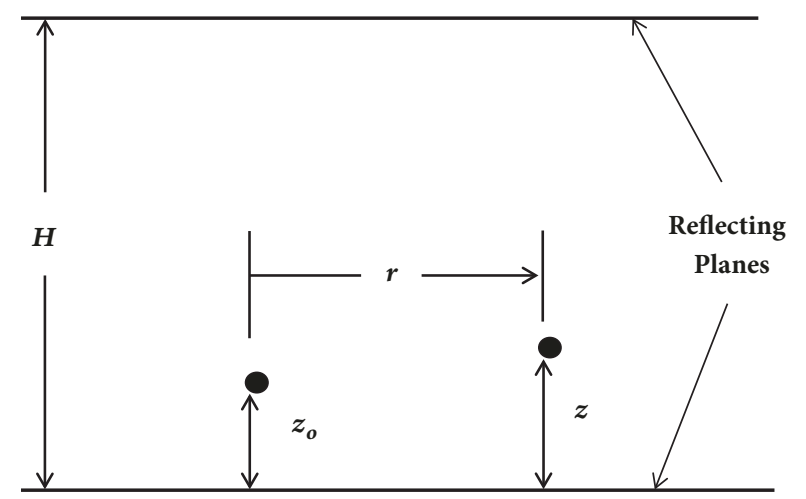

FIgURE 1: Schematic of a diametrical cross section of the acoustic space.

This system transfer function in [11] leads directly to an input/output ordinary differential equation which serves as the basis for defining the state variables and determining the state equations. For the acoustic space of the current paper, the Euler-Maclaurin sum formula must be directly applied to the time domain form of the Green's function infinite series as is done in [8]. As previously indicated, that direct application of the Euler-Maclaurin sum formula to the time domain form of the Green's function infinite series [8] leads to an integral of a radical function that has no exact analytical solution for providing polynomial type functions in the Laplace transform domain. Thus, in this paper, this radical function is first approximated with a combination of two exponential types to provide a suitable simple system overall analytical transfer function for proceeding to the input/output ordinary differential equation that is used to develop the state space model. Typical noise inputs of impulse, harmonic, and random each including high frequency character up to $3000 \mathrm{~Hz}$. are used to compare the performance state space model with the performance of many terms in the series model. Two extreme numerical cases of plates separation $H=9 \mathrm{~m}$. (30 ft.) for a typical industrial area to $H=0.3 \mathrm{~m}$. (1 $\mathrm{ft}$.) for a typical tight-fitting enclosure such as for a diesel engine system are presented numerically.

\section{Mathematical Model}

2.1. Euler-Maclaurin Representation. A schematic of the diametrical cross section in the z-r plane for a three-dimensional acoustic space is shown in Figure 1.

The acoustic field between two perfectly reflecting parallel planes can be represented as an infinite sum of images in the Laplace transform domain (see $[12,13]$ ).

$$
\begin{aligned}
4 \pi G(r, z, s)= & \frac{e^{-(s / c) \sqrt{r^{2}+\left(z+z_{o}\right)^{2}}}}{\sqrt{r^{2}+\left(z+z_{o}\right)^{2}}}+\frac{e^{-(s / c) \sqrt{r^{2}+\left(z-z_{o}\right)^{2}}}}{\sqrt{r^{2}+\left(z-z_{o}\right)^{2}}} \\
& +\sum_{k=1}^{\infty} \frac{e^{-(s / c) \sqrt{r^{2}+\left(2 k H+z+z_{o}\right)^{2}}}}{\sqrt{r^{2}+\left(2 k H+z+z_{o}\right)^{2}}}
\end{aligned}
$$




$$
\begin{aligned}
& +\sum_{k=1}^{\infty} \frac{e^{-(s / c) \sqrt{r^{2}+\left(2 k H-z-z_{o}\right)^{2}}}}{\sqrt{r^{2}+\left(2 k H-z-z_{o}\right)^{2}}} \\
& +\sum_{k=1}^{\infty} \frac{e^{-(s / c) \sqrt{r^{2}+\left(2 k H+z-z_{o}\right)^{2}}}}{\sqrt{r^{2}+\left(2 k H+z-z_{o}\right)^{2}}} \\
& +\sum_{k=1}^{\infty} \frac{e^{-(s / c) \sqrt{r^{2}+\left(2 k H-z+z_{o}\right)^{2}}}}{\sqrt{r^{2}+\left(2 k H-z+z_{o}\right)^{2}}}
\end{aligned}
$$

where $\mathrm{H}$ is the distance between the planes.

$\mathrm{z}_{\mathrm{o}}$ and $\mathrm{z}$ are the source and receiver position between the planes.

$\mathrm{r}$ is the distance $\sqrt{x^{2}+y^{2}}$ in the $\mathrm{x}, \mathrm{y}$ plane from source to receive parallel to the planes.

$\mathrm{c}$ is the speed of sound $(345 \mathrm{~m} / \mathrm{s}, 1130 \mathrm{f} / \mathrm{s})$.

$\mathrm{G}(\mathrm{r}, \mathrm{z}, \mathrm{s})$ is the Laplace transform of the Green's function $\mathrm{g}(\mathrm{x}, \mathrm{y}, \mathrm{z}, \mathrm{t})$ for the wave equation

$$
\begin{gathered}
\nabla_{x y z}^{2} g(x, y, x, t)-\frac{1}{c^{2}} \frac{\partial^{2} g(x, y, z, t)}{\partial t^{2}} \\
=-\delta(\mathrm{x}) \delta(\mathrm{y}) \delta\left(\mathrm{z}-\mathrm{z}_{\mathrm{o}}\right) \delta(\mathrm{t})
\end{gathered}
$$

In [14], the form of the Euler-Maclaurin sum formula that transforms an infinite series to a closed form expression with a remainder $\mathrm{R}$ is used here. It is given by

$$
\begin{array}{r}
\sum_{k=1}^{\infty} f_{k}=\int_{1}^{\infty} f(\mu) d \mu+\left(\frac{1}{2}\right)[f(1)+f(\infty)]+R \\
R=O\left\{\left(\frac{1}{2}\right)[f(1)+f(\infty)]\right\}
\end{array}
$$

In [8] the Euler-Maclaurin sum formula in (3) is applied to the four infinite series in (1), the inverse Laplace is applied, and the following closed form solution of the Green's function in the time domain is obtained.

$$
\begin{aligned}
& 4 \pi g(r, z, t)=\frac{1}{r_{1}} \delta\left(t-\frac{r_{1}}{c}\right)+\frac{1}{r_{2}} \delta\left(t-\frac{r_{2}}{c}\right) \\
& +\frac{1}{2 H \sqrt{t^{2}-(r / c)^{2}}}\left\{U\left(t-\frac{r_{3}}{c}\right)+U\left(t-\frac{r_{4}}{c}\right)\right. \\
& \left.+U\left(t-\frac{r_{5}}{c}\right)+U\left(t-\frac{r_{6}}{c}\right)\right\}+\sum_{i=3}^{6} \frac{1}{2 r_{i}} \delta\left(t-\frac{r_{i}}{c}\right) \\
& +\sum_{i=3}^{6} R_{i} \quad R_{i}=O\left\{\frac{1}{2 r_{i}} \delta\left(t-\frac{r_{i}}{c}\right)\right\}
\end{aligned}
$$

where $U$ is the Heaviside step function and $\mathrm{O}$ is an order at most.

$$
\begin{aligned}
& r_{1}=\sqrt{r^{2}+\left(z+z_{o}\right)^{2}} \\
& r_{2}=\sqrt{r^{2}+\left(z-z_{o}\right)^{2}} \\
& r_{3}=\sqrt{r^{2}+\left(2 H+z+z_{o}\right)^{2}} \\
& r_{4}=\sqrt{r^{2}+\left(2 H+z-z_{o}\right)^{2}} \\
& r_{5}=\sqrt{r^{2}+\left(2 H-z-z_{o}\right)^{2}} \\
& r_{6}=\sqrt{r^{2}+\left(2 H-z+z_{o}\right)^{2}}
\end{aligned}
$$

The Green's function is composed of discrete delta functions $\delta$ and a group of four dispersion-like terms with Heaviside step function $U$ that come from the integral in the EulerMaclaurin sum formula.

For purposes of showing the concept of a state space representation, (4) is simplified by using $z=z_{o}=0$ without the loss of generality. The Green's function in (4) is expressed as $g(r, t)=2 \pi g$ and is given by

$$
\begin{aligned}
& g(r, t)= \frac{1}{r} \delta\left(t-t_{R}\right)+\frac{1}{\sqrt{r^{2}+(2 H)^{2}}} \delta\left(t-t_{o}\right) \\
&+\frac{1}{H \sqrt{t^{2}-R^{2}}} U\left(t-t_{o}\right) \\
& \text { where } t_{R}=\frac{r}{c}, t_{o}=\frac{\sqrt{r^{2}+(2 H)^{2}}}{c}
\end{aligned}
$$

and the remainders $R$ are considered small enough to be neglected as shown in [8].

The main reverberation term is the third term in (6) and monotonically decreases from a maximum of $H_{s}=c / 2 H^{2}$ at $t=t_{o}$ to 0 at $t=\infty$. It is conceivable to reason that the sum of several to many exponentials would provide a reasonably accurate fit over a reasonable range of $t$. Each exponential would increase the system order by one and thus several to many significant states would result. However, the goal of this approximate model is to provide a more simple compact form with just two exponential terms while still providing a significantly influential model, albeit with a lower number of states. Reasonable for this acoustic system may be defined as providing good enough accuracy for use in numerical simulations for various type of inputs, for use in input estimates (see [11]), and for use in some control applications. In a frequency description of reverberant sound in a large industrial space, it is shown in [15] how a function composed of two exponentials, one with constant coefficient and one with time varying coefficient, could be used to accurately represent multiple model floor and ceiling reflections. A similar model is used here and is given by 


$$
\begin{aligned}
& \frac{1}{H \sqrt{t^{2}-R^{2}}} U\left(t-t_{o}\right) \\
& \cong\left[H_{\mathrm{s}} \mathrm{e}^{-\mathrm{a}\left(\mathrm{t}-\mathrm{t}_{\mathrm{o}}\right)}+\mathrm{B}\left(t-t_{o}\right) \mathrm{e}^{-\mathrm{b}\left(\mathrm{t}-\mathrm{t}_{\mathrm{o}}\right)}\right] U\left(t-t_{o}\right)
\end{aligned}
$$

where parameters $a, b$, and $B$ are determined to provide a good fit over the time range from $t_{o}$ to some suitable value $T$. Goodness of fit and suitability are defined here as providing a reasonably accurate comparison of the behavior of the developed state space model with the infinite series of image in (1) for a range of practical input sources. The input sources used here are an impact type rapidly decaying exponential source, a single high frequency harmonic input, and a broadband input generated with a modified multifrequency Schroeder phased harmonic sequence as shown in [11]. B:

A four-part procedure is given for determining $a, b$, and

(1)

Definine time $\bar{t}=t-t_{o}$,

$$
\text { where } \bar{T}=T-t_{o}
$$

consider a time $\bar{T}_{c}<\bar{T}$ such that

$$
\begin{gathered}
\text { for } 0<\bar{t}<\bar{T}_{c}, \\
\left(y_{a}=H_{s} \mathrm{e}^{-\mathrm{a} \bar{t}}\right)>\left(y_{E M}=\frac{1}{H \sqrt{\bar{t}^{2}-R^{2}}}\right)
\end{gathered}
$$

$$
\begin{aligned}
\text { and at } \bar{t} & =\bar{T}_{c}, \\
\left(y_{a}=H_{s} \mathrm{e}^{-\mathrm{a} \bar{T}_{c}}\right) \cdot= & \left(y_{E M}=\frac{1}{H \sqrt{\bar{T}_{c}^{2}-R^{2}}}\right) \\
\text { This gives } a & =\frac{\ln \left((c / 2 H) \sqrt{\bar{T}_{c}^{2}-R^{2}}\right)}{\bar{T}}
\end{aligned}
$$

(2)

For the value of a from part 1 , define $y_{b}=B \bar{t} e^{-b \bar{t}}$

$$
=y_{a}-y_{E M}=H_{\mathrm{s}} \mathrm{e}^{-\mathrm{a} \overline{\mathrm{t}}}-\frac{1}{H \sqrt{\bar{t}^{2}-R^{2}}}
$$

The maximim value of $y_{b}$ occurs for $\frac{d y_{b}}{d \bar{t}}=0$

giving $\bar{t}_{\max }=\frac{1}{b}$

which gives $y_{b \operatorname{macx}}=-\left(\frac{B}{b}\right) e^{-1}$

(3)

$$
\text { Using } y_{b}=\operatorname{Max}\left|H_{\mathrm{s}} \mathrm{e}^{-\mathrm{a} \overline{\mathrm{t}}}-\frac{1}{H \sqrt{\bar{t}^{2}-R^{2}}}\right| \text { from part 2, }
$$

$\bar{t}_{\max }$ is determined numerically and the last two equations in (9) give

$$
b=\frac{1}{\bar{t}_{\max }}
$$

$$
\text { and } B=-e y_{b \max } b
$$

(4) Steps (1), (2), and (3) give a good set of values for $a$, $b$, and $B$. These values are then used as the initial set for an optimization procedure to obtain a better set of values. The optimization function to minimize is given by

$$
\begin{aligned}
& F(a, b, B)=\int_{t_{o}}^{T} \mid \frac{1}{H \sqrt{t^{2}-R^{2}}} \\
& -\left[H_{\mathrm{s}} \mathrm{e}^{-\mathrm{a}\left(\mathrm{t}-\mathrm{t}_{\mathrm{o}}\right)}+\mathrm{B}\left(t-t_{o}\right) \mathrm{e}^{-\mathrm{b}\left(\mathrm{t}-\mathrm{t}_{\mathrm{o}}\right)}\right] \mid d t
\end{aligned}
$$

for a time $T$ suitable for covering the primary part of the Green function response.
To demonstrate this, two numerical cases of the $r, H$ set representing somewhat of an extreme range are presented. The first is for $H=3 \mathrm{~m}$. (10 ft.), $r=9 \mathrm{~m}$. (30 ft.) which may apply to a large work space or open plan off area and the second is for $H=0.3 \mathrm{~m}$. (1 $1 \mathrm{ft}.), r=0.9 \mathrm{~m}$. ( $3 \mathrm{ft}$.) which may apply to the inside of a tight-fitting machine enclosure. Both cases are selected so that the ratio of $r$ to $H$ is the same value of 3 .

Table 1 gives the results for the two cases. Units for the table are based on $H$ and $r$ in $\mathrm{ft}$.

Figures 2 and 3 give a comparison of the two sides of (7) for cases 1 and 2, respectively. In the figures, the left hand side of (7) (which comes from the closed form representation using the Euler-Maclaurin sum formula on the infinite series) 
TABLE 1: Values for (7).

\begin{tabular}{lcccccccccc}
\hline $\mathrm{H}$ & $\mathrm{r}$ & $\mathrm{a}$ & $\mathrm{t}_{\max }$ & $\mathrm{b}$ & $\mathrm{B}$ & $\mathrm{T}$ & $\mathrm{T}_{\mathrm{c}}$ & $\mathrm{a}_{\text {opt }}$ & $\mathrm{b}_{\text {opt }}$ & $\mathrm{B}_{\text {opt }}$ \\
\hline 10 & 30 & 2.4 & .097 & 15.3 & -156 & 3 & 2 & 6.1 & 13.6 & -102 \\
1 & 3 & 23.9 & .0097 & 152.8 & $-1.56 \times 10^{5}$ & .3 & .2 & 61 & 136 & $-1.02 \times 10^{5}$ \\
\hline
\end{tabular}

is defined as yem, the first term in the right side of (7) is defined as $y 1$, the second term in the right side of (7) is defined as y2, and the total approximation is defined as yapp $=y 1+y 2$. The horizontal axes in the figures are the time $\bar{t}=t-t_{o}$. The figures clearly show the good accuracy for the most important early part of the approximation with still reasonable accuracy for the latter part of the transformation. The trend is consistent through the entire time. The match in the time scale of the right hand plots is less because the exponential approximation selected for the simple state space model contains two basic terms. Additional accuracy for longer time could be achieved for the right hand time frame by using more exponential type terms but would defeat the purpose of having a simple model. Practically all of the audible sound heard occurs before this latter time frame. The exponential input results are discussed with respect to accuracy for early and later parts of the time response relative to the peak response. Peak response is used as the baseline for errors because of the importance near the peak.

2.2. State Space Representation. Starting with the Green's function of (6) with the right side approximation of (7) and using $g(t)$ to represent $g(r, t)$,

$$
\begin{aligned}
g(t)= & \frac{\delta\left(t-t_{R}\right)}{r}+\frac{\delta\left(t-t_{o}\right)}{c t_{o}} \\
& +\left[H_{s} e^{-a\left(t-t_{o}\right)}+B\left(t-t_{o}\right) e^{-b\left(t-t_{o}\right)}\right] U\left(t-t_{o}\right)
\end{aligned}
$$

Applying the Laplace Transform $G(s)=\int_{0}^{\infty} g(r, t) e^{-s t} d t$ gives

$$
G(s)=\frac{e^{-s t_{R}}}{r}+\frac{e^{-s t_{o}}}{c t_{o}}+\frac{H_{s} e^{-s t_{o}}}{s+a}+\frac{B e^{-s t_{o}}}{(s+b)^{2}}
$$

Cross multiplying by $(s+a)(s+b)^{2}=s^{3}+(2 b+a) s^{2}+\left(b^{2}+\right.$ $2 a b) s+a b^{2}$ gives

$$
\begin{aligned}
& {\left[s^{3}+(2 b+a) s^{2}+\left(b^{2}+2 a b\right) s+a b^{2}\right] G(s)} \\
& =\left[s^{3}+(2 b+a) s^{2}+\left(b^{2}+2 a b\right) s+a b^{2}\right] \\
& \quad \cdot\left(\frac{e^{-s t_{R}}}{r}+\frac{e^{-s t_{o}}}{c t_{o}}\right)=H_{s}\left(s^{2}+2 b s+b^{2}\right) e^{-s t_{o}} \\
& \quad+B(s+a) e^{-s t_{o}}
\end{aligned}
$$

To simplify notation, the delta functions with time shifts are defined as

$$
\begin{aligned}
& \bar{\delta}_{R}=\delta\left(t-t_{R}\right), \\
& \bar{\delta}_{t o}=\delta\left(t-t_{o}\right) .
\end{aligned}
$$

Applying the inverse Laplace transform gives a third-order differential equation for $g(t)$.

$$
\begin{aligned}
\dddot{g}(t) & +(2 b+a) \ddot{g}(t)+\left(b^{2}+2 a b\right) \dot{g}(t)+\left(a b^{2}\right) g(t) \\
= & \frac{1}{r}\left[\ddot{\bar{\delta}}_{R}+(2 b+a) \ddot{\bar{\delta}}_{R}+\left(b^{2}+2 a b\right) \dot{\bar{\delta}}_{R}+a b^{2} \bar{\delta}_{R}\right] \\
& \cdot \frac{1}{c t_{o}}\left[\ddot{\bar{\delta}}_{t o}+(2 b+a) \ddot{\bar{\delta}}_{t o}+\left(b^{2}+2 a b\right) \dot{\bar{\delta}}_{t o}+a b^{2} \bar{\delta}_{t o}\right] \\
& +H_{s} \ddot{\bar{\delta}}_{t o}+\left(2 H_{s} b+B\right) \dot{\bar{\delta}}_{t o}+\left(H_{s} b^{2}+B a\right) \bar{\delta}_{t o}
\end{aligned}
$$

Collecting terms gives

$$
\begin{aligned}
\dddot{g}( & +(2 b+a) \ddot{g}(t)+\left(b^{2}+2 a b\right) \dot{g}(t)+\left(a b^{2}\right) g(t) \\
= & \frac{1}{r}\left[\ddot{\bar{\delta}}_{R}+(2 b+a) \ddot{\bar{\delta}}_{R}+\left(b^{2}+2 a b\right) \dot{\bar{\delta}}_{R}+a b^{2} \bar{\delta}_{R}\right] \\
& \cdot \frac{1}{c t_{o}} \frac{\ddot{\delta_{t o}}}{c t_{o}}+\left(\frac{2 b+a}{s}\right) \ddot{\bar{\delta}}_{t o} \\
& +\left(\frac{b^{2}+2 a b}{c t_{o}}+2 H_{s} b+B\right) \dot{\bar{\delta}}_{t o} \\
& +\left(\frac{a b^{2}}{c t_{o}}+H_{s} b^{2}+B a\right) \bar{\delta}_{t o}
\end{aligned}
$$

The input, output equation is obtained by substituting output $y$ for $g(t)$ and input $\bar{u}$ for $\bar{\delta}$.

Define the coefficients as

$$
\begin{aligned}
& a_{2}=2 b+a \\
& a_{1}=b^{2}+2 a b \\
& a_{0}=a b^{2} \\
& p_{o}=\frac{a b^{2}}{r} \\
& p_{1}=\frac{b^{2}+2 a b}{r} \\
& p_{2}=\frac{2 b+a}{r} \\
& p_{3}=\frac{1}{r} \\
& q_{o}=\left(\frac{a b^{2}}{c t_{o}}+H_{s} b^{2}+B a\right)
\end{aligned}
$$



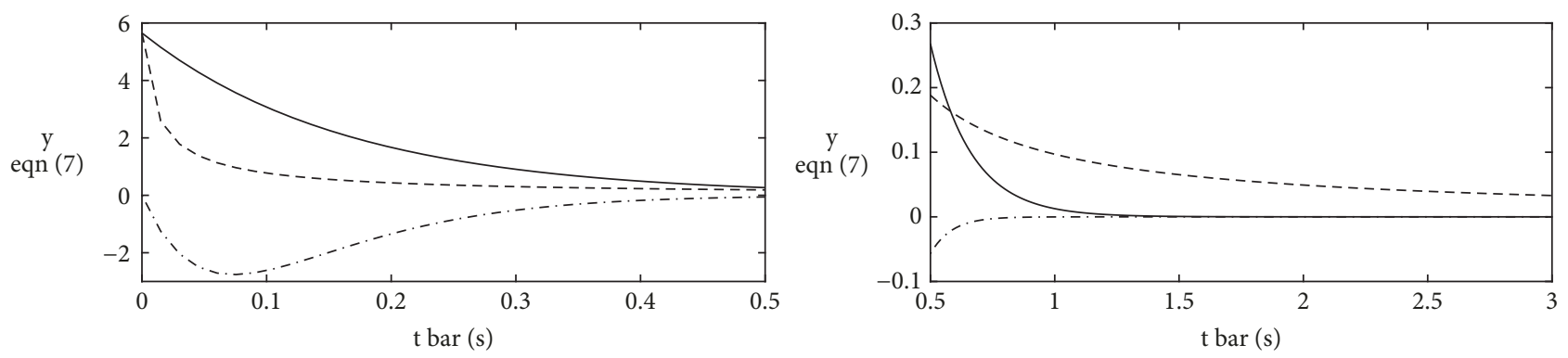

$$
\begin{aligned}
& --- \text { yem } \\
& -\mathrm{yl}
\end{aligned}
$$$$
--- \text { yem }
$$$$
-\mathrm{y} 1
$$$$
-y^{2}
$$
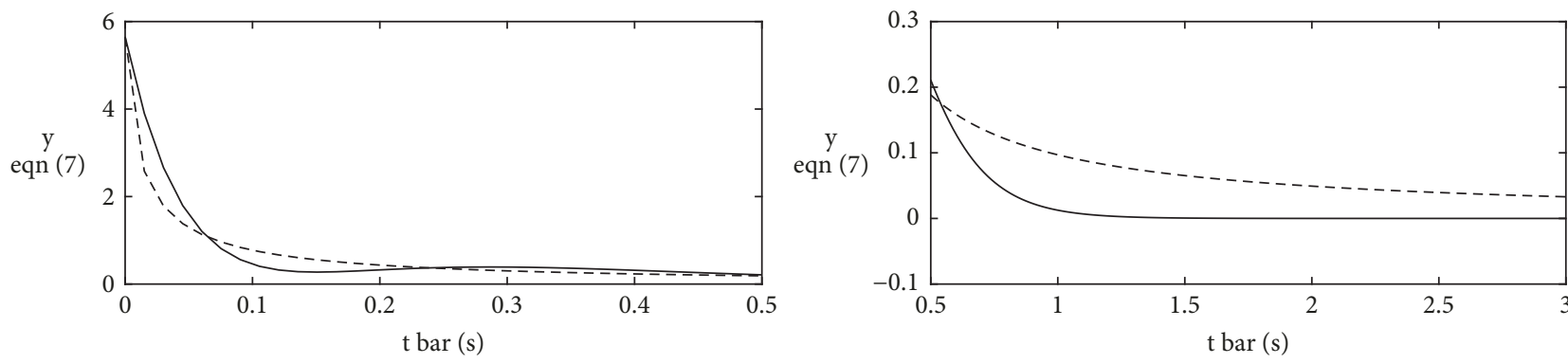

$\begin{array}{ll}--- & \text { yem } \\ - & \text { yapp }\end{array}$

--- yem

- yapp

Figure 2: Euler-Maclaurin (7) comparison, Case 1.
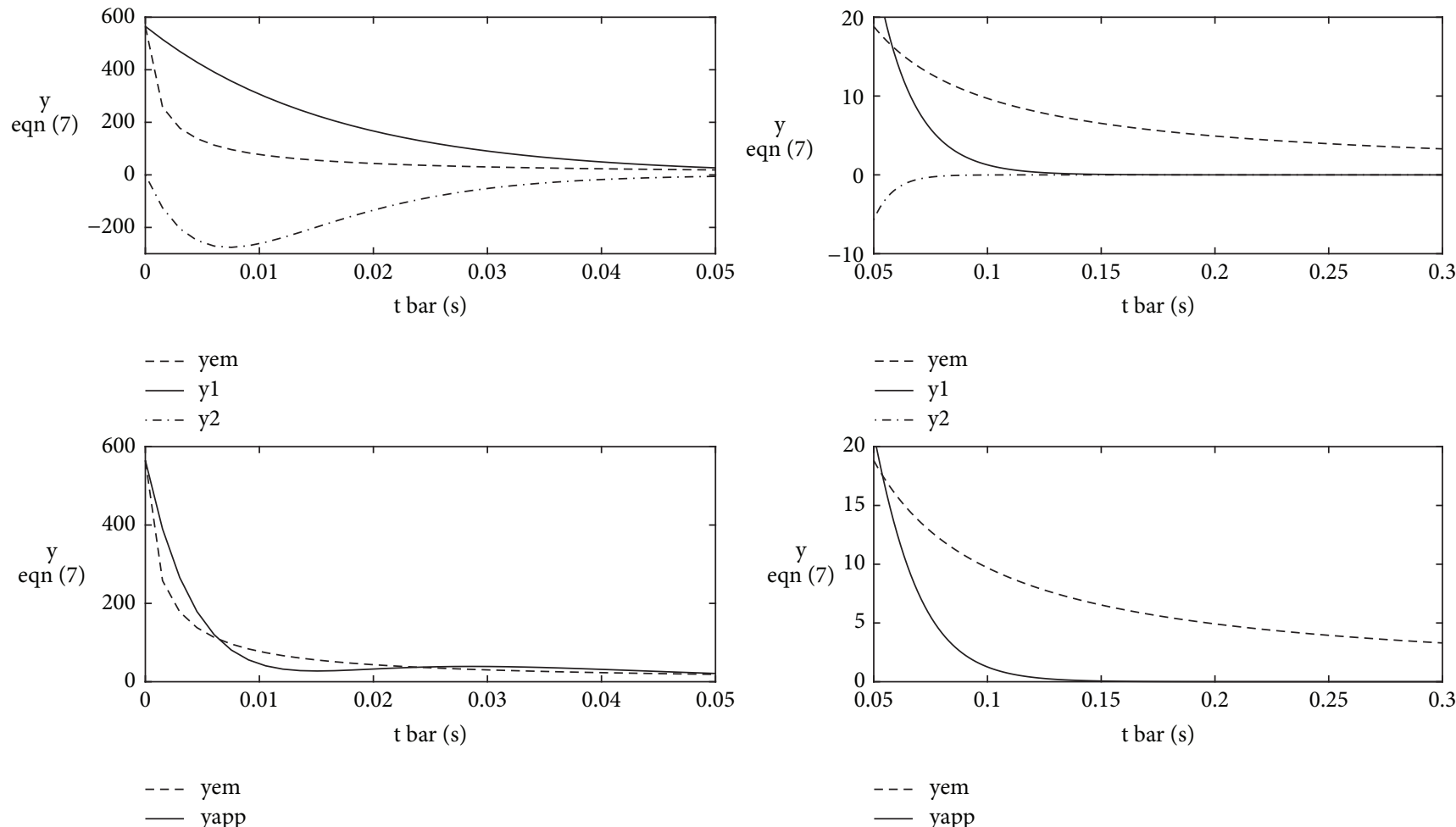

Figure 3: Euler-Maclaurin (7) comparison, Case 2. 


$$
\begin{aligned}
& q_{1}=\frac{b^{2}+2 a b}{c t_{o}}+2 H_{s} b+B \\
& q_{2}=\frac{2 b+a}{c t_{o}}+H_{s} \\
& q_{3}=\frac{1}{c t_{o}}
\end{aligned}
$$

The input, output form of (17) is given by

$$
\begin{aligned}
\dddot{y}+a_{2} \ddot{y}+a_{1} \dot{y}+a_{o} y= & p_{3} \ddot{\bar{u}}_{R}+p_{2} \ddot{\bar{u}}_{R}+p_{1} \dot{\bar{u}}_{R}+p_{o} \bar{u}_{R} \\
& +q_{3} \ddot{\bar{u}}_{t o}+q_{2} \ddot{\bar{u}}_{t o}+q_{1} \dot{\bar{u}}_{t o} \\
& +q_{o} \bar{u}_{t o}
\end{aligned}
$$

Three states are defined:

$$
\begin{aligned}
& x_{1}=y \\
& x_{2}=\dot{y}-\beta_{o} \bar{u}_{R}-\alpha_{o} \bar{u}_{t o} \\
& x_{3}=\dot{x}_{2}-\beta_{1} \bar{u}_{R}-\alpha_{1} \bar{u}_{t o}
\end{aligned}
$$

where $\alpha_{o}, \beta_{o}, \alpha_{1}$, and $\beta_{1}$ are coefficients to be determined in terms of system parameters $a, b, B, r, H, c$, and $t_{o}$.

The first state equation is determined by taking the derivative of the first state definition and substituting $\dot{y}$ from the second state definition. It is given by

$$
\dot{x}_{1}=x_{2}+\beta_{o} \bar{u}_{R}+\alpha_{o} \bar{u}_{t o}
$$

The second state equation is determined by first taking the derivative of the second state definition which brings a $\ddot{y}$ term.

$$
\dot{x}_{2}=\ddot{y}-\beta_{o} \dot{\bar{u}}_{R}-\alpha_{o} \dot{\bar{u}}_{t o}
$$

Next substituting $\dot{x}_{2}$ from the third state definition into (22) and solving for $\ddot{y}$ gives

$$
\ddot{y}=x_{3}+\beta_{1} \bar{u}_{R}+\alpha_{1} \bar{u}_{t o}+\beta_{o} \dot{\bar{u}}_{R}+\alpha_{o} \dot{\bar{u}}_{t o}
$$

The second state equation is then given by

$$
\begin{aligned}
\dot{x}_{2}= & x_{3}+\beta_{1} \bar{u}_{R}+\alpha_{1} \bar{u}_{t o}+\beta_{o} \dot{\bar{u}}_{R}+\alpha_{o} \dot{\bar{u}}_{t o}-\beta_{o} \dot{\bar{u}}_{R} \\
& -\alpha_{o} \dot{\bar{u}}_{t o} \\
\dot{x}_{2}= & x_{3}+\beta_{1} \bar{u}_{R}+\alpha_{1} \bar{u}_{t o}
\end{aligned}
$$

The third state equation is determined by first taking the derivative of the third state definition and using the derivative of (22) which brings in a $\dddot{y}$ term.

$$
\begin{aligned}
\dot{x}_{3} & =\ddot{x}_{2}-\beta_{1} \dot{\bar{u}}_{R}-\alpha_{1} \dot{\bar{u}}_{t o} \\
& =\dddot{y}-\beta_{o} \ddot{\bar{u}}_{R}-\alpha_{o} \ddot{\bar{u}}_{t o}-\beta_{1} \dot{\bar{u}}_{R}-\alpha_{1} \dot{\bar{u}}_{t o}
\end{aligned}
$$

Solving (25) for $\dddot{y}$ and equating it to $\dddot{y}$ from the input, output form of (19) gives

$$
\begin{aligned}
\dot{x}_{3}+ & \beta_{1} \dot{\bar{u}}_{R}+\alpha_{1} \dot{\bar{u}}_{t o}+\beta_{o} \ddot{\bar{u}}_{R}+\alpha_{o} \ddot{\bar{u}}_{t o} \\
= & -a_{2} \ddot{y}-a_{1} \dot{y}-a_{o} y+p_{3} \ddot{\bar{u}}_{R}+p_{2} \ddot{\bar{u}}_{R}+p_{1} \dot{\bar{u}}_{R} \\
& +p_{o} \bar{u}_{R}+q_{3} \ddot{\bar{u}}_{t o}+q_{2} \ddot{\bar{u}}_{t o}+q_{1} \dot{\bar{u}}_{t o}+q_{o} \bar{u}_{t o}
\end{aligned}
$$

Substituting $y, \dot{y}$, and $\ddot{y}$ from above and collecting terms gives

$$
\begin{aligned}
\dot{x}_{3}+ & \beta_{1} \dot{\bar{u}}_{R}+\alpha_{1} \dot{\bar{u}}_{t o}+\beta_{o} \ddot{\bar{u}}_{R}+\alpha_{o} \ddot{\bar{u}}_{t o}+a_{2} x_{3}+a_{2} \beta_{1} \bar{u}_{R} \\
& +a_{2} \alpha_{1} \bar{u}_{t o}+a_{2} \beta_{o} \dot{\bar{u}}_{R}+a_{2} \alpha_{o} \dot{\bar{u}}_{t o}+a_{1} x_{2}+a_{1} \beta_{o} \bar{u}_{R} \\
& +a_{1} \alpha_{0} \bar{u}_{t o}+a_{o} x_{1} \\
= & p_{3} \stackrel{\bar{u}}{R}+p_{2} \ddot{\bar{u}}_{R}+p_{1} \dot{\bar{u}}_{R}+p_{o} \bar{u}_{R}+q_{3} \ddot{\bar{u}}_{t o}+q_{2} \ddot{\bar{u}}_{t o} \\
& +q_{1} \dot{\bar{u}}_{t o}+p_{o} \bar{u}_{t o}
\end{aligned}
$$

where $p$ 's and $q$ 's are defined in (18).

To determine the coefficients $\alpha_{o}, \beta_{o}, \alpha_{1}$, and $\beta_{1}$ used in the state definitions, like terms in (27) are combined and the coefficients of the six $u, \dot{u}$, and $\ddot{u}$ terms on each side of the equation are set equal to give

$$
\begin{aligned}
& \bar{u}_{R} \longrightarrow a_{2} \beta_{1}+a_{1} \beta_{o}=p_{o} \\
& \bar{u}_{t o} \longrightarrow a_{2} \alpha_{1}+a_{1} \alpha_{o}=q_{o} \\
& \dot{\bar{u}}_{R} \longrightarrow \beta_{1}+a_{2} \beta_{o}=p_{1} \\
& \dot{\bar{u}}_{t o} \longrightarrow \alpha_{1}+a_{2} \alpha_{o}=q_{1} \\
& \ddot{\bar{u}}_{R} \longrightarrow \beta_{o}=p_{2} \\
& \ddot{\bar{u}}_{t o} \longrightarrow \alpha_{o}=q_{2}
\end{aligned}
$$

$\beta_{o}$ and $\alpha_{o}$ are determined by the third equation in (28).

$\beta_{1}$ and $\alpha_{1}$ are determined from the first or second equation combined with the third in (28) and are given by

$$
\begin{aligned}
& \beta_{1}=p_{1}-a_{2} p_{2} \\
& \alpha_{1}=q_{1}-a_{2} q_{2}
\end{aligned}
$$

Substituting from (28) and (29) into (26) gives the third state equation

$$
\begin{aligned}
\dot{x}_{3}= & -a_{o} x_{1}-a_{1} x_{2}-a_{2} x_{3}+\left(p_{o}-a_{2} \beta_{1}-a_{1} \beta_{o}\right) \bar{u}_{R} \\
& +\left(q_{o}-a_{2} \alpha_{1}-a_{1} \alpha_{o}\right) \bar{u}_{t o}+p_{3} \dddot{\bar{u}}_{R}+q_{3} \dddot{\bar{u}}_{t o}
\end{aligned}
$$


The finite state space differential equation is given by

$$
\dot{\mathbf{x}}=\mathbf{A x}+\mathbf{B u} \quad \mathbf{u}=\left[\begin{array}{c}
\bar{u}_{R} \\
\bar{u}_{t o} \\
\dddot{\bar{u}}_{R} \\
\dddot{\bar{u}}_{t o}
\end{array}\right] \mathbf{A}=\left[\begin{array}{ccc}
0 & 1 & 0 \\
0 & 0 & 1 \\
-a_{o} & -a_{1} & -a_{2}
\end{array}\right] \mathbf{B}=\left[\begin{array}{cccc}
\beta_{o} & \alpha_{o} & 0 & 0 \\
\beta_{1} & \alpha_{1} & 0 & 0 \\
p_{o}-a_{2} \beta_{1}-a_{1} \beta_{0} & q_{o}-a_{2} \alpha_{1}-a_{1} \alpha_{o} & p_{3} & q_{3}
\end{array}\right]
$$

The system in (31) contains delays in the input. The output $y$ of interest is the first state $x_{1}$. The state matrix $\mathbf{A}$ has a canonical form with the third-order characteristic equation

$$
\lambda^{3}+a_{2} \lambda^{2}+a_{1} \lambda+a_{0}=0 .
$$

\section{Evaluation of State Space Model}

3.1. Example Inputs. Three common types of noise inputs are evaluated: impulse, pure tone, and random.

3.1.1. Exponential Input. To simulate an impulsive (impact) type of noise, a simple decaying exponential function with decay rate $\alpha$ is used

$$
u(t)=e^{-\alpha t}
$$

3.1.2. Pure Tone Input. To simulate a continuous pure tone type of noise, a single harmonic sine function with radian frequency $\omega$ is used.

$$
u(t)=\sin (\omega t)
$$

3.1.3. Broadband Input. To simulate a broadband type of input that can provide a response over a typical range of noise frequencies from 100 to $3000 \mathrm{~Hz}$, a multifrequency Schroeder phased harmonic sequence used in [11] with random amplitudes and variable phases is given by

$$
\begin{aligned}
& u(t)=\sum_{k=1}^{N_{k}} A_{k} \sin \left(k \omega_{o} t+\varphi_{k}\right) \\
& \varphi_{k}=\varphi_{k-1}-2 \pi \sum_{j=1}^{k-1} \frac{A_{j}^{2}}{\sum_{k=1}^{N_{k}} A_{k}^{2}}
\end{aligned}
$$

where $\omega_{o}=2 \pi(100 \mathrm{~Hz}), N_{k}=30$ to provide an input from $100 \mathrm{~Hz}$ to $3000 \mathrm{~Hz}$ and $0 \leq A_{k} \leq 1$ via a uniformly distributed random number generator. This input is randomlike in time (as used in [11]) and provides a suitable model for a broadband multifrequency input for evaluation in this paper.

3.2. Numerical Comparison of Series and State Space Model. The numerical solution to the state space model of differential equations (31) is determined with a special fourth-order Runge-Kutta method modified to efficiently handle the delay inputs. This solution is compared to the solution from a direct sum of three hundred terms in a convolution of the infinite series in (1) with the input. From (1), the series representation of Green's function is given by

$$
\begin{aligned}
& g(r, t)=2 \pi g \\
& =\frac{1}{r} \delta\left(t-\frac{r}{c}\right) \\
& \quad+2 \sum_{k=1}^{\infty} \frac{1}{\sqrt{r^{2}+(2 k H)^{2}}} \delta\left(t-\frac{\sqrt{r^{2}+(2 k H)^{2}}}{c}\right)
\end{aligned}
$$

Convolution with the input $u(t)$ gives the output $\mathrm{y}(\mathrm{t})$ for comparison

$$
\begin{aligned}
& y(r, t)=\int_{o}^{t} g(r, \tau) u(t-\tau) d \tau=\int_{o}^{t}\left[\frac{1}{r} \delta\left(\tau-\frac{r}{c}\right)\right. \\
& \left.\quad+2 \sum_{k=1}^{\infty} \frac{1}{\sqrt{r^{2}+(2 k H)^{2}}} \delta\left(\tau-\frac{\sqrt{r^{2}+(2 k H)^{2}}}{c}\right)\right] \\
& \cdot u(t-\tau) d \tau
\end{aligned}
$$

Using the sampling property of the delta function, the series output is given by

$$
\begin{aligned}
& y(r, t)=\frac{1}{r} u\left(t-\frac{r}{c}\right) U\left(t-\frac{r}{c}\right) \\
& +2 \sum_{k=1}^{\infty} \frac{1}{\sqrt{r^{2}+(2 k H)^{2}}} u\left(t-\frac{\sqrt{r^{2}+(2 k H)^{2}}}{c}\right) \\
& \cdot U\left(t-\frac{\sqrt{r^{2}+(2 k H)^{2}}}{c}\right)
\end{aligned}
$$

For computations, 300 terms in the sum provide a very accurate series solution for comparing the state space solution. 
For the Euler-Maclaurin closed form approximation, convolution of input $u$ and the Green function in (6) give

$$
\begin{gathered}
y(r, t)=\int_{o}^{t}\left[\frac{1}{r} \delta\left(\tau-t_{R}\right)+\frac{1}{\sqrt{r^{2}+(2 H)^{2}}} \delta\left(\tau-t_{o}\right)\right. \\
\left.+\frac{1}{H \sqrt{\tau^{2}-R^{2}}} U\left(\tau-t_{o}\right)\right] u(t-\tau) d \tau
\end{gathered}
$$

Using the sampling property of the delta function, the EulerMaclaurin closed form output is given by

$$
\begin{aligned}
y(r, t)= & \frac{1}{r} u\left(t-\frac{r}{c}\right) U\left(t-\frac{r}{c}\right) \\
& +\frac{1}{\sqrt{r^{2}+(2 H)^{2}}} u\left(t-t_{0}\right) U\left(t-t_{o}\right) \\
& +\int_{o}^{t}\left[\frac{1}{H \sqrt{\tau^{2}-R^{2}}} U\left(\tau-t_{o}\right)\right] u(t-\tau) d \tau
\end{aligned}
$$

where the integral in (40) is solved numerically for each time increment.

Both systems in Table 1 are used as relevant examples comparing the state space output to the series output for each of the three types of inputs.

Figures 4 and 5 give the comparison for the exponential input. Input exponential parameters $\alpha=1$ and $\alpha=5$ are used to show a range for a typical slow impulse noise and a typical fast impulse noise, respectively. In the figures, the "state space" output is the first state for the solution to the finite state space model of (31) which is obtained from the Euler-Maclaurin closed form Green function. The "closed form" output is from (40) which is obtained from direct integration of the Euler-Maclaurin closed form solution. The "series" output is from (38), a summation of the infinite series for $N=300$ values. For the case of $H=3 \mathrm{~m}$. (10 ft.) in Figure 4 , the state space model is very accurate. For the case of $H=0.3 \mathrm{~m}$. (1 $\mathrm{ft}$.) in Figure 5, the state space model is very accurate for the early part of transient and follows an accurate decaying trend with values a little lower than the series solution. For both cases, the larger value of $\alpha$ gives the best accuracy. This indicates that since larger values of $\alpha$ tend to simulate more of true impulse response which defines natural system behavior, the state space model may be well suited for control applications. The closed form model provides similar accuracy as the state space for both cases and is included to show how well the state space Euler-Maclaurin model agrees with the state space model derived from it.

Table 2 gives a numerical assessment of the accuracy in Figures 4 and 5 for key parts of the response. For an exponential input used in to model for a practical impact type sound input, the important parts of the transient response are near the ascent from zero to the maximum value, the maximum value at the time, and the descent from the maximum value to a time that is several times the peak time $t_{\text {peak. }}$ As the time increases to this area and beyond, the effect
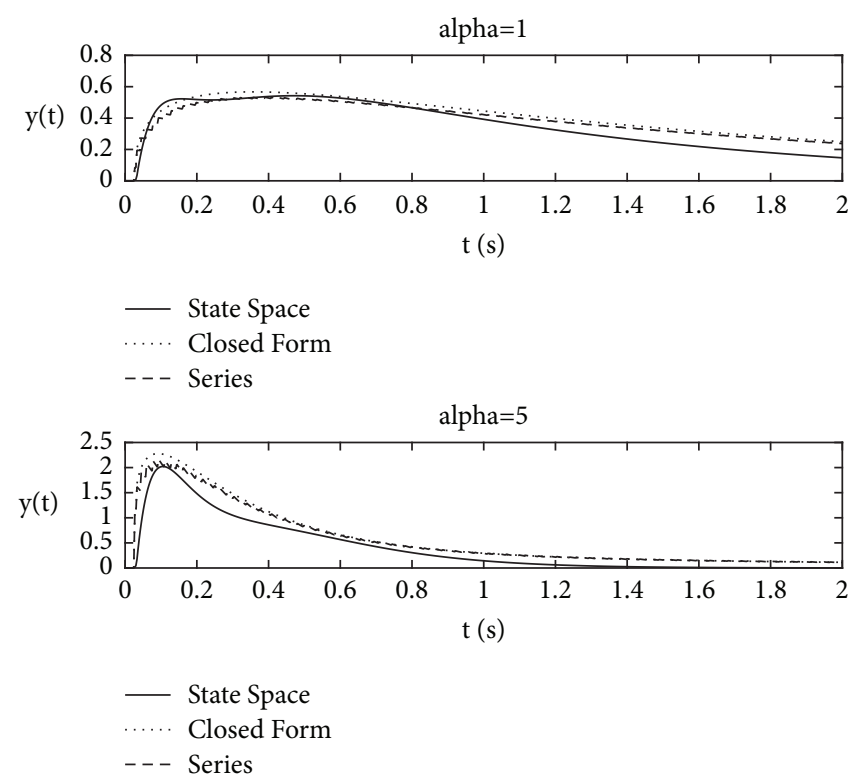

FIgURE 4: Exponential input response, Case 1.
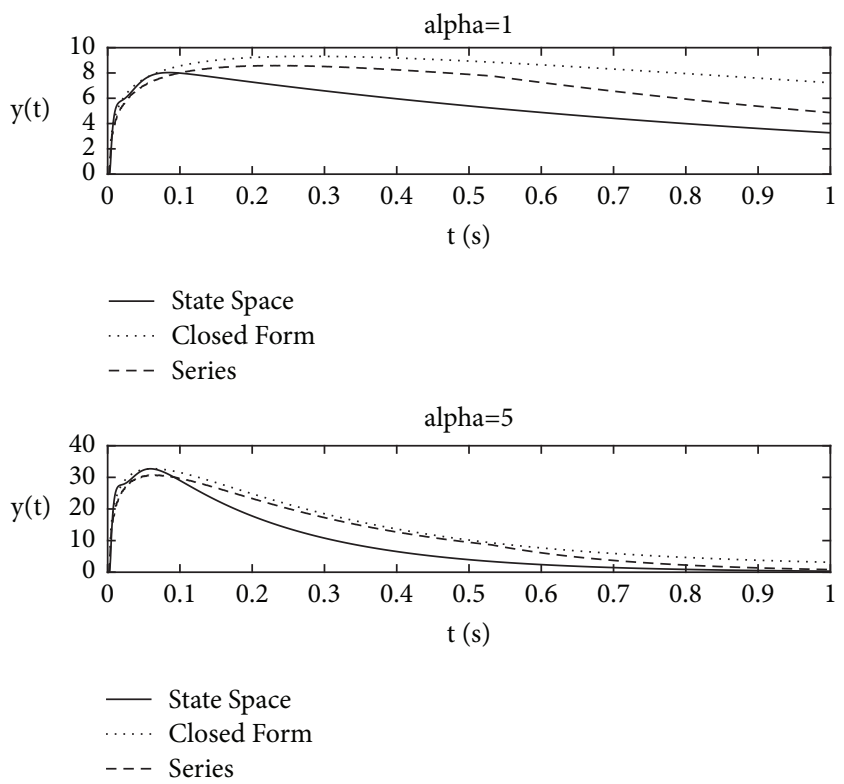

FIgURE 5: Exponential input response, Case 2.

from this type of impact input on a system becomes much less important. Many practical noise sources are essentially a series of impacts which are space apart enough such that a periodic frequency associated with it is low, usually below about $300 \mathrm{~Hz}$. The noise level and character one audibly hears or measures is usually broadband and primarily comes from a single impact each time it hits and is amplified by how many hits per second fit into a specified time frame for an equivalent time average level. A common example is impact noise in industrial environments from various and many sources of rigid body contacts occurring frequently and randomly causing the familiar audible sound of myriad of superimposed distinct bangs. The effects of these many 
similar impacts per second provide a broadband mid to high frequency spectrum that are the main contributors to the overall dBA level. Another example is the interaction of cooling system propeller fan blades with still air and/or guide vanes. Generally the fundamental blade passage harmonic frequency is below $300 \mathrm{~Hz}$, dominates the level of higher harmonics, and is only a small contributor to the overall dBA noise. The primary contributor is the turbulent impulses that occur at each interaction. As the industrial impact noise sounding like a series of bangs, each pulse is audible as broadband turbulence. The effects of these many similar impulses per second provide a broadband mid to high frequency spectrum that are the main contributors to the overall $\mathrm{dBA}$ level. There are other examples such as combustion engine pressure cycling that may fall into this class. For this class of noise sources, the audible sound is characteristically that of a particular occurrence of the impact/impulse heard over and over again.

To reflect the importance of the response at and near maximum, the percent error between state space and series responses that is used in Table 2 is defined as

$$
\% \text { Error }=\left|\frac{\mathrm{y}_{\text {series }}-y_{\text {state space }}}{y_{\text {max series }}}\right| 100
$$

The percent errors for the exponential input are mostly in the $1 \%$ to $7 \%$ range with a few higher ones in a $12 \%$ to $26 \%$ range occurring for case 2 during the descent from the peak. This level of error may be considered reasonable for the intent of the model. One reason for large errors for case 2 during the $2^{\text {nd }}$ descent may be the use of the simple twoterm approximation for yapp which leads to it being a little less accurate for higher time areas as seen in Figures 3 and 4. However, the important monotonically decreasing trend is still present in this higher time range.

Figures 6 and 7 give the comparison for a harmonic input of typical acoustic range frequencies $1000 \mathrm{~Hz}$. and $2000 \mathrm{~Hz}$. In the figures, the "state space" output is the first state for the solution to the finite state space model of (31) which is obtained from the Euler-Maclaurin closed form Green function. The "series" output is from (38), a summation of the infinite series for $N=300$ values. For both $H$ value cases, the amplitudes of the two models are off by about $50 \%$, while the phases between the two models are much closer.

Table 3 gives a numerical assessment of amplitude and phase errors defined by

$$
\begin{aligned}
\% \text { Amplitude Error } & =\frac{\| y_{\max \text { series }}|-| y_{\text {max state }} \mid}{\left|y_{\text {max of series or state }}\right|} 100 \\
\% \text { Amplitude Ratio } & =\left|\frac{y_{\max \text { state }}}{y_{\max \text { series }}}\right| 100 \text { or } \\
& =\left|\frac{y_{\max \text { series }}}{y_{\max \text { state }}}\right| 100
\end{aligned}
$$

$$
\% \text { Phase Ratio }=\frac{\mid \text { Time difference of Peaks } \mid}{\text { Period }}
$$

The amplitude errors for the harmonic inputs are much larger than those for the exponential input's amplitude errors
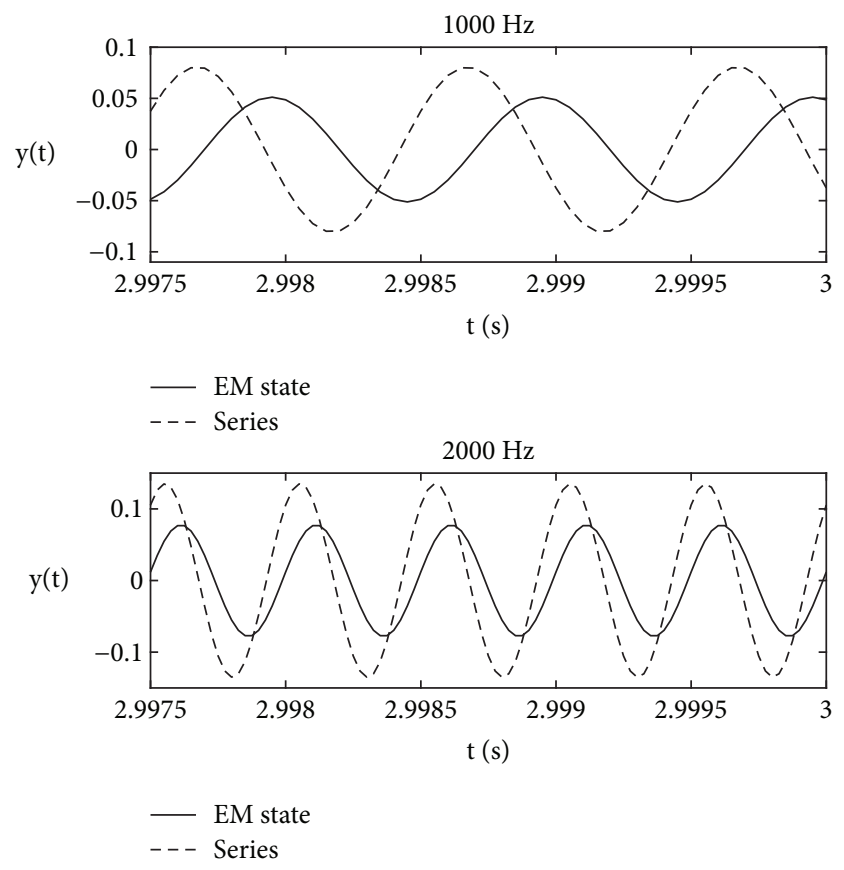

FIgURE 6: Harmonic input response, Case 1.

for the key part of the exponential input. One reason for larger amplitude errors may be the fact that harmonic inputs can potentially cause standing waves to be more prevalent between the two reflecting plane, especially for case $2(H=$ $.3 \mathrm{~m})$ where the wavelengths $(.34 \mathrm{~m}$ for $1000 \mathrm{~Hz}$ and $.17 \mathrm{~m}$ for $2000 \mathrm{~Hz}$ ) are near full and half wavelengths of $H$. Also noted is that the amplitude errors for case 1 are about one-half of those for case 2, while the phase errors for case 2 are about one-half of those for case 1 .

Figure 8 shows a typical input trace $u(t)$ for the multifrequency Schroeder phased harmonic sequence for $N_{k}=30$ frequencies in a frequency range from $f_{o}=100 \mathrm{~Hz}$. to $N_{k} f_{o}=$ $3000 \mathrm{~Hz}$. The randomness in time is seen primarily via peak amplitude and pulse width. The randomness in frequency is less with a tendency of the highest peaks in a range from $300 \mathrm{~Hz}$. to $500 \mathrm{~Hz}$. and the lower peaks in in a range from $2000 \mathrm{~Hz}$. to $2500 \mathrm{~Hz}$. These ranges are indicated on the figure.

Figure 9 gives the comparison for multifrequency Schroeder phased harmonic sequence. In the figures, the "state space" output is the first state for the solution to the finite state space model of (31) which is obtained from the Euler-Maclaurin closed form Green function. The "series" output is from (38), a summation of the infinite series for $N=300$ values. For the case of $H=3 \mathrm{~m}$. (10 ft.), there is very close agreement in that an equivalent dominant frequency of approximately $1100 \mathrm{~Hz}$ from eleven pulses over the 0.01 seconds shown is evident in both the Series and EMstate responses. A similar comparison is seen for the case of $H=$ $0.3 \mathrm{~m}$. ( $1 \mathrm{ft}$.) where there is very close agreement in that an equivalent dominant frequency of approximately $2600 \mathrm{~Hz}$ from twenty-six pulses over the 0.01 seconds shown is evident in both the Series and EMstate responses. For both cases, the series amplitudes are larger than the EMstate amplitudes, 
TABLE 2: Numerical assessment of exponential inputs results in Figures 4 and 5.

\begin{tabular}{|c|c|c|c|c|}
\hline Error Description & Case $1 \alpha=1$ & Case $1 \alpha=5$ & Case $2 \alpha=1$ & Case $2 \alpha=5$ \\
\hline \multicolumn{5}{|l|}{ Ascent to $1^{\text {st }}$ state peak } \\
\hline Time Range (s) & $0-.15$ & $0-.092$ & $0-.077$ & $0-.061$ \\
\hline Min to Max Error (\%) & $0-16.7$ & $4.2-9.3$ & $1.3-5.5$ & $1.3-7.1$ \\
\hline Average (\%) & 5.5 & 5.8 & 4.1 & 4.6 \\
\hline \multicolumn{5}{|l|}{ State Peak } \\
\hline Time (s), $t_{\text {peak }}$ & .15 & .092 & .077 & .061 \\
\hline Peak Error (\%) & 14.6 & 2.8 & 2.7 & 5.4 \\
\hline \multicolumn{5}{|c|}{$1^{\text {st }}$ Descent to an additional $4 t_{\text {peak }}$ peak intervals } \\
\hline Time Range (s) & $.15-.75$ & $.092-.46$ & $.077-.38$ & $.061-.31$ \\
\hline Min to Max Error (\%) & $0-4.2$ & $2.8-15.7$ & $0-23.6$ & $0-20.2$ \\
\hline Average (\%) & 1.2 & 6.8 & 14.0 & 12.3 \\
\hline \multicolumn{5}{|c|}{$2^{\text {nd }}$ Descent to an additional $4 t_{\text {peak }}$ more peak intervals } \\
\hline Time Range (s) & $.75-1.4$ & $.46-.83$ & $.38-.69$ & $.31-.66$ \\
\hline Min to Max Error (\%) & $1.0-10.4$ & 2.8-5.7 & 23.6-27.7 & 16.2-18.9 \\
\hline Average (\%) & 4.6 & 2.6 & 26.4 & 17.5 \\
\hline
\end{tabular}
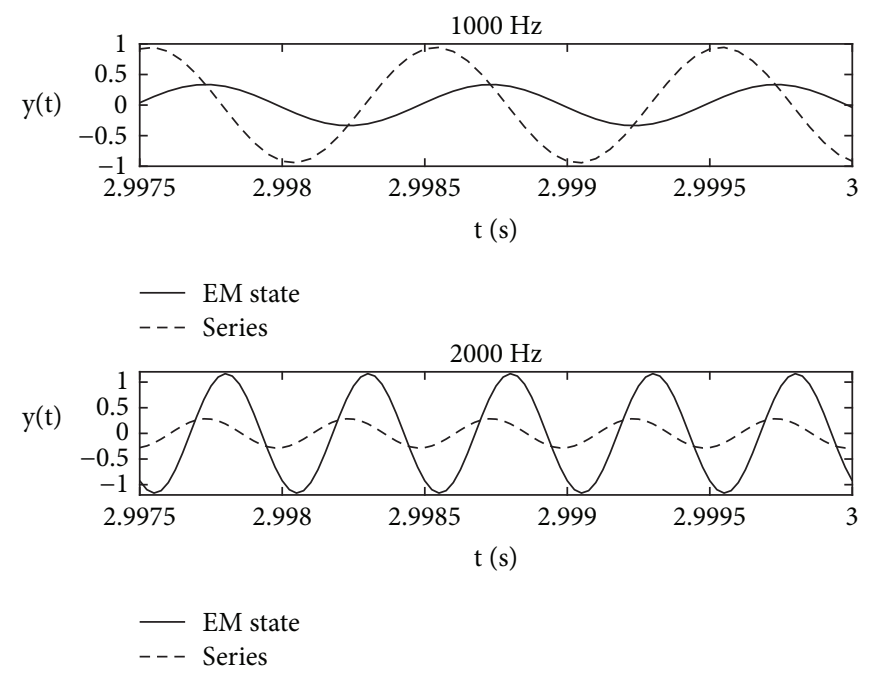

FIGURE 7: Harmonic input response, Case 2.

while the EMstate pulse widths are larger than the series pulse widths. Using a combination of like frequency in the comparison as well as similar area under a typical pulse to provide a measure that can be expected to significantly affect sound level and character, the accuracy of the EMstate model may be considered reasonable for its intended uses as described previously. The work in [11] uses a similar state space derived model for estimating the random input to a plate via one output measurement and an observer based on the state model. This also may be a suitable area of application for the acoustic state model.

Table 4 shows a numerical comparison associated with the measures above.

The comparison of the two primary indicators of dominant frequency (which has essentially a $0 \%$ error between Series and EMstate) and the pulse area (of which the EMstate is close to $70 \%$ of the series) may be considered to provide a reasonable amount of accuracy for the intended use of the EM state model.

The following summary of the results presented in the previous comparison curves and tables provides clarity of the scope and the applicability of the state space model for analysis and control. For the exponential input as a model of impact noise, the model may be considered accurate enough for use in both the analysis and control of single and multiple impact sources of the type discussed where the behavior up to, at, and just after the peak response is primarily responsible for the audible noise. For the harmonic input, the model may be considered reasonably accurate for analysis to estimate the response amplitude for larger plate separation distances. However, for the harmonic input, even though phase errors are less than amplitude errors, the response has only a single phase and the level of this phase error shown is considered too high for providing confidence in 


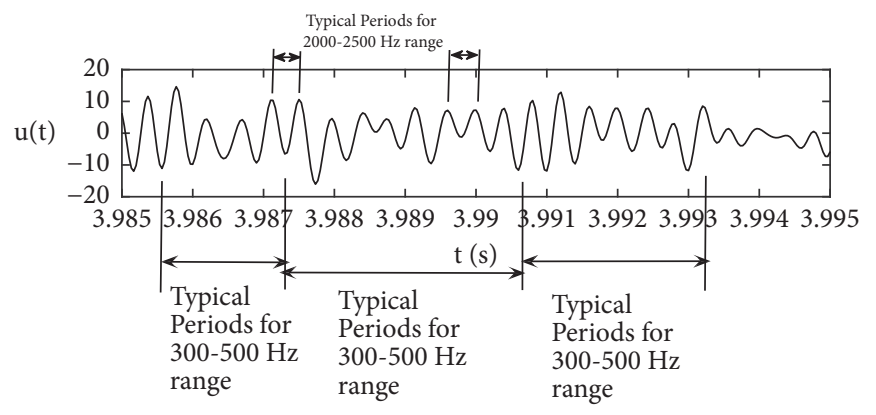

FIGURE 8: Broadband Schroeder input trace $u(t)$.
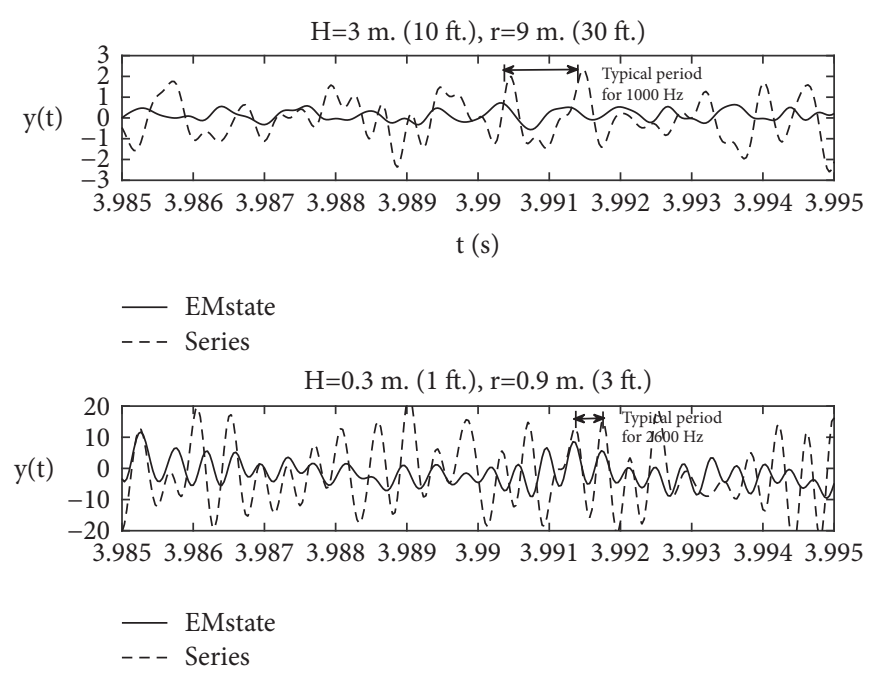

Figure 9: Broadband Schroeder input response.

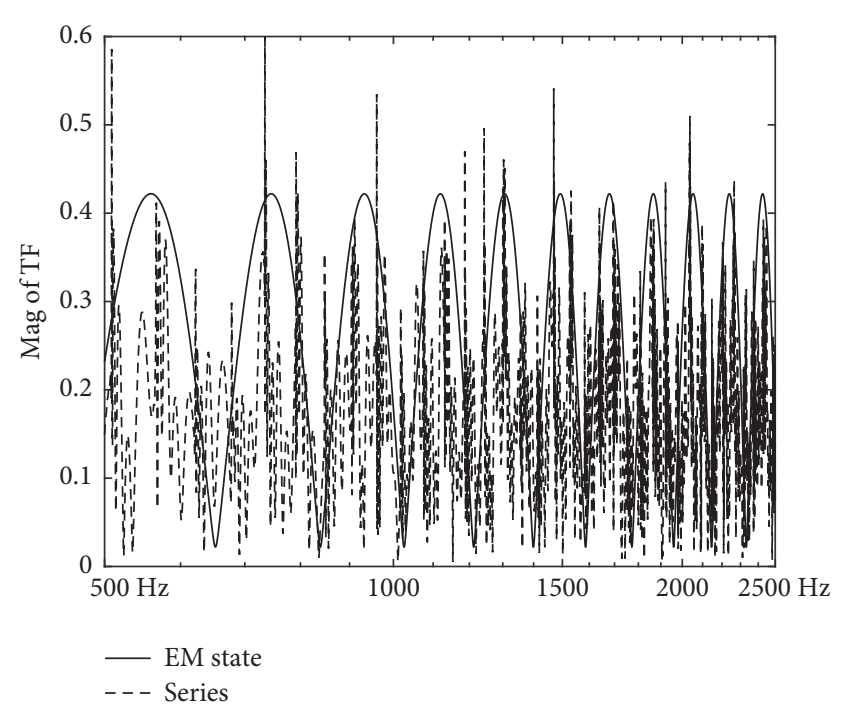

FIGURE 10: Transfer function comparison.

the application of common control schemes to reduce levels from pure tone noise sources. For the Schroeder random like input model, the reasonable accuracy obtained for both response frequency occurrence and pulse area indicates that the state space model may be used with a reasonable amount of confidence in the analysis of overall noise level and character. Now since the Schroeder is not exactly random but composed of a series of pure tones with various phases and amplitudes, its makeup gives preference to selected frequencies as clearly shown in Figure 8. But Figure 9 shows that the response has varying phase errors on the order of magnitude as the harmonic phase. In the realm of noise issues such as in manufacturing, transportation, and ventilation, single impacts, repeated impacts, and broadband random type acoustic sources are generally much more prevalent than pure tone sources. The numerical results from the exponential and the Schroeder input representations of these types of sources indicate that the scope of the closed form Euler-Maclaurin state space model is reasonably accurate for analysis and control in these areas. The next section gives a frequency response method used by the author in [9] for testing how a Euler-Maclaurin approximate closed form system model can be tested against the corresponding series solution in a feedback control scheme for a purely random input disturbance of white noise which may be used to represent a broadband noise source. 


\section{Frequency Response}

Figure 10 is a comparison of the magnitude of the transfer function for input $u(t)$ to output $y(t)$ obtained by substituting $s=j \omega$ in (1) and the transfer function for the Euler-Maclaurin state space model is determined by applying the Laplace transform to (31) and obtaining an expression for the transfer function.

$$
\begin{aligned}
\mathbf{X}(s) & =(s \mathbf{I}-\mathbf{A})^{-1} \mathbf{B}(s) \mathbf{U}(s) \\
\mathbf{Y}(s) & =\mathbf{C X}(s) \\
\mathbf{C} & =\left[\begin{array}{lll}
1 & 0 & 0
\end{array}\right] \\
\mathbf{U}(s) & =\left[\begin{array}{c}
U_{R}(s) \\
U_{t o}(s) \\
s^{3} U_{R}(s) \\
s^{3} U_{t o}(s)
\end{array}\right]=U(s)\left[\begin{array}{c}
e^{-s R} \\
e^{-s t o} \\
s^{3} e^{-s R} \\
s^{3} e^{-s t o}
\end{array}\right] \\
& =U(s) \mathbf{Z}(s) \\
T F(s) & =\frac{Y(s)}{U(s)}=\mathbf{C}(s \mathbf{I}-\mathbf{A})^{-1} \mathbf{B}(s) \mathbf{Z}(s) \\
T F m a g & =|T F(j \omega)|
\end{aligned}
$$

The overall magnitudes across frequencies are comparable. The differences in shape comparison may be expected from the nature of the two models. The series is expected to contain many sharp narrow bandwidth spikes of varying amplitude and frequency as it is obtained from an infinite sum all of the images between two rigid plates. The EM state model is obtained from a closed form expression via the EulerMaclaurin sum formula and derived using the two exponential approximate expression in (7). The finite EM state transfer function result indicates that the many frequencies due to images are consolidated into several frequencies of the same magnitude but with bandwidths increasing with frequency, indicating that the EM state model gives a look of more damping than the series model.

To test the use of the EM state model for feedback control of a broadband random noise source, a feedback control scheme is shown in Figure 11, where $F_{d}(s)$ is the noise source input considered as a disturbance.

It is a model based output feedback control scheme requiring a measurement of the system output. This method was tested in [9] with a white noise disturbance and was shown to be useful for reducing white noise amplitudes in a two-dimensional acoustical space with an EM transfer function obtained directly from the closed form EM approximate function derived in that paper. The same method is applied here using an EM transfer function obtained from the Euler-Maclaurin state space model of (31) and (33). It is used to determine the controller transfer function required

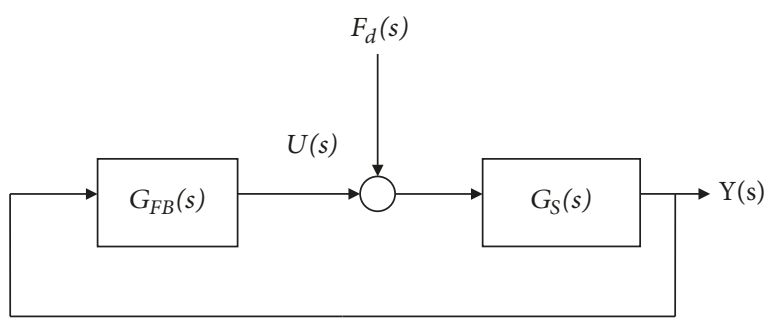

FIGURE 11: Feedback control scheme for broadband noise disturbance.

to provide a desired output over a broad range of frequencies. The closed loop system transfer function is given by

$$
T F(s)=\frac{Y(s)}{F_{d}(s)}=\frac{G_{S}(s)}{1+G_{S}(s) G_{F B}(s)},
$$

where $G_{F B}(s)$ is the feedback controller transfer function. For the controller design, consider a desired closed loop transfer function $T F_{\text {des }}(s)$ and use the finite Euler-Maclaurin transfer function $G_{E M}(s)$ obtained in (43) in place of $G_{s}(s)$ equation $(44)$ to solve for $G_{F B}(s)$. The finite controller transfer function is then given by

$$
G_{F B}(s)=\frac{G_{E M}(s)-T F_{d}(s)}{G_{E M}(s) T F_{d}(s)} .
$$

The actual closed loop system transfer function from (44) is given by

$$
\begin{aligned}
& T F_{\text {actual }}(s)=\frac{Y(s)}{F_{d}(s)} \\
& =\frac{G_{S}(s)}{1+G_{S}(s)\left[\left(G_{E M}(s)-T F_{d}(s)\right) / G_{E M}(s) T F_{d}(s)\right]} .
\end{aligned}
$$

If one uses the actual plant transfer function (which has many terms) as the control model plant transfer function (i.e., $G_{E M}(s)=G_{S}(s)$ ), in the controller equation (45), the actual closed loop system output transfer function from (46) would equal the desired transfer function (i.e., $T F_{\text {actual }}(s)=$ $\left.T F_{\text {des }}(s)\right)$. One has the advantage of using the finite EM state transfer function (with only one term for higher order modes) for the plant transfer function in the model based controller design to give the finite feedback controller transfer function of (45).

For a white noise broad representation of a broadband disturbance, the desired transfer function magnitude $\left|T F_{\text {des }}(s)\right|$ is a constant. Figures 12 and 13 give the results of the magnitude of the closed loop transfer function $\left(\left|T F_{\text {actual }}(s)\right|\right.$ from (46)) relative to $\left|T F_{\text {des }}(s)\right|$ and the uncontrolled series $\left|G_{s}(s)\right|$. Figure 12 is for $\left|T F_{\text {des }}(s)\right|=0.06$ and Figure 13 is for $\left|T F_{\text {des }}(s)\right|=0.02$. Additionally, the figures give the magnitude of the controller transfer function (see (45)) for each $\left|T F_{\text {des }}(s)\right|$ value to demonstrate the gain and character of the controller magnitude $\left|G_{F B}(s)\right|$.

For both Figures 12 and 13, the control drives the highly variable magnitude of the series transfer function over frequency to the desired closed loop magnitude with very 
TABLE 3: Numerical assessment of harmonic input results in Figures 6 and 7.

\begin{tabular}{lcccc}
\hline Frequency $(\mathrm{Hz})$ & Case & \% Amplitude Error & \% Amplitude Ratio & \% Phase Error \\
\hline 1000 & 1 & 28 & 72 & 28 \\
2000 & 1 & 40 & 30 & 11 \\
1000 & 2 & 61 & 39 & 17 \\
2000 & 2 & 73 & 27 & 7 \\
\hline Average & $1 \& 2$ & 50 & 50 & 15 \\
\hline
\end{tabular}

TABLE 4: Numerical assessment of Schroeder input results in Figure 9.

\begin{tabular}{|c|c|c|c|c|}
\hline Measure Description & Case 1 State & Case 1 Series & Case 2 State & Case Series \\
\hline \multicolumn{5}{|l|}{ Equivalent Dominant Frequency } \\
\hline Approximate Number of Pulses across $0.01 \mathrm{~s}$. & 11 & 11 & 26 & 26 \\
\hline $\begin{array}{l}\text { Equivalent } \\
\text { Frequency }(\mathrm{Hz})\end{array}$ & 1100 & 1100 & 2600 & 2600 \\
\hline Error $(\%)$ & 0 & 0 & 0 & 0 \\
\hline \multicolumn{5}{|l|}{ Amplitude across .01 s. } \\
\hline Amplitude Range & $.43-1.0$ & $.71-2.2$ & $3.8-12.3$ & $5.7-19.8$ \\
\hline Average Amplitude & .67 & 1.66 & 7.5 & 14.1 \\
\hline Average pulse width (s) & .00062 & .00039 & .00031 & .00023 \\
\hline Average area per pulse & .00043 & .00064 & .0022 & .0032 \\
\hline Ratio of State pulse area to Series pulse area & $\begin{array}{c}\text { Case } 1 \\
67 \%\end{array}$ & & $\begin{array}{c}\text { Case } 2 \\
69 \%\end{array}$ & \\
\hline $\begin{array}{l}\text { Phase Difference across .01 s } \\
\text { (Qualitative) }\end{array}$ & $\begin{array}{c}\text { Case } 1 \\
\text { Only some match }\end{array}$ & & $\begin{array}{c}\text { Case } 2 \\
\text { Close match for most } \\
\text { pulses }\end{array}$ & \\
\hline
\end{tabular}

small fluctuations over the frequency range. The controller required to accomplish this consists of a steady gain plus a smaller variable oscillation over frequency. From Figure 10, the system uncontrolled series transfer function gives a frequency average of approximately 0.2. In Figure 12, the control reduces it to the 0.06 desired value. The controller steady gain is approximately 17.5. In Figure 13, the control reduces it to the 0.02 desired value. The controller steady gain is approximately 50 . As expected, the higher the required reduction, the higher the controller gain. This provides some confidence that a similar type of control may be useful for a Schroeder type disturbance that is configured in such a way as to provide a more random like input that may serve as good model for broadband noise similar to white noise but not exactly having the constant desired transfer function.

\section{Conclusions}

A simple low order but full finite space model is developed that naturally includes a broadband frequency range and essentially all the terms required for a convergent infinite series in the Green's function representation of the acoustic field between reflecting planes. The model includes the use of a closed form analytical expression for Green's function obtained from the application of the Euler-Maclaurin sum formula. A radical function in the closed form Green's function is approximated with a combination of two exponential types to provide a suitable system overall analytical transfer function for proceeding to the input/output ordinary differential equation that is used to develop the state space model. The resulting state model is a third-order system with time delayed inputs and naturally includes all modes (images) of reflection between the reflecting plates. No modal truncation is considered. The model is tested by comparing its solution to a baseline series solution for three common noise inputs for a broad range of frequencies up to $3000 \mathrm{~Hz}$. Numerical results comparing the state model and the series solution are provided for small and large distances between reflecting plates typical of acoustic environments in industrial or commercial spaces to tight-fitting noise enclosures. The issues of accuracy are extensively examined from time simulation responses and key error calculations in a table for each of the three inputs. Tables 2, 3, and 4 provide a numerical assessment of exponential, harmonic, and broadband inputs results, respectively for cases 1 and 2 with various \% error and $\%$ ratio values for amplitudes, frequencies, and phases as required to customize for the specific input type. The intent is to show how the simple approximate model compares with the series model not as always a specific $y(t)$ value comparison for every time instant but as the behavior of $y(t)$ along the time scale for several issues key to the specific input. A test for use of the model for feedback control of random noise is included. Overall, the results show the scope of the 

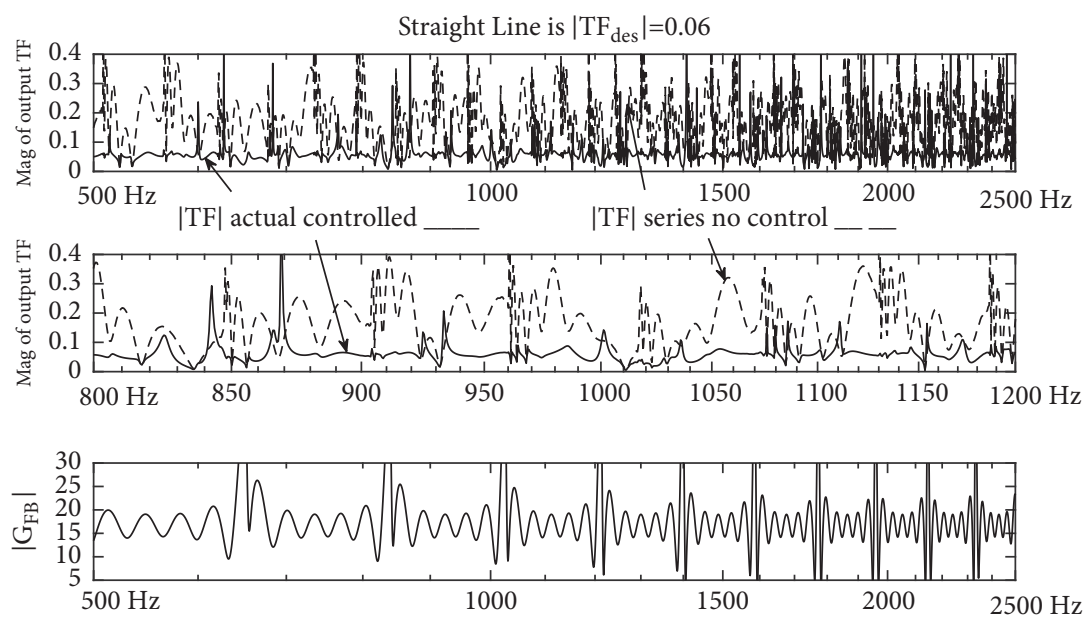

FIGURE 12: Feedback control transfer functions for $\left|\mathrm{TF}_{\text {desired }}\right|=0.06$.
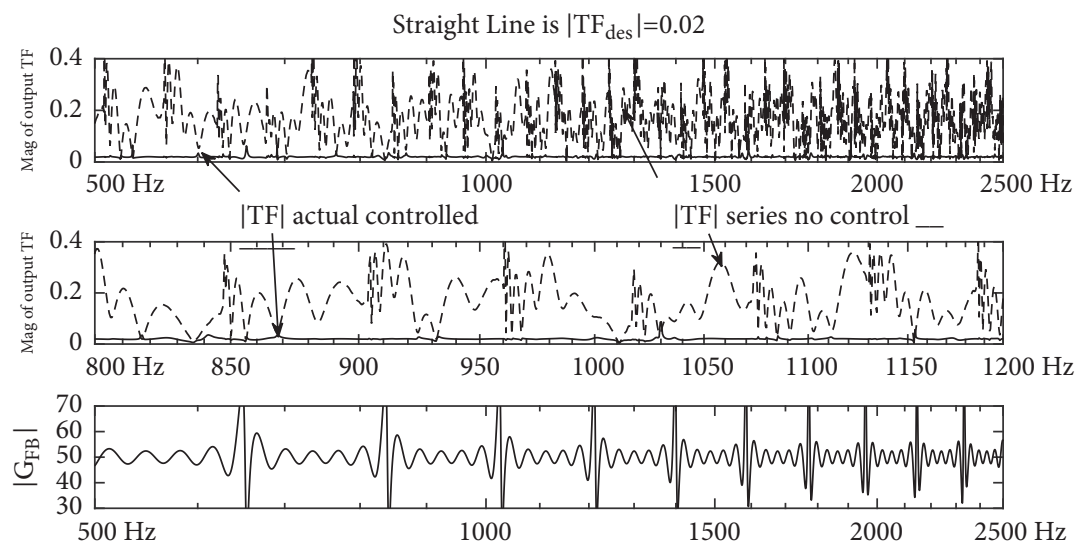

FIGURE 13: Feedback control transfer functions for $\left|\mathrm{TF}_{\text {desired }}\right|=0.02$.

approximate model's use in analysis and control. The model gives reasonably good accuracy and confidence for the use of a simple finite state model in state space noise analysis and control applications for impact and random type noise which are prevalent in practice. For a pure tone noise, the accuracy may be reasonably good for approximate analysis of output magnitude but the phase differences in the outputs of series and state models are significant enough to provide concern for its use in controlling single frequency sources.

\section{Conflicts of Interest}

The author declares that there are no conflicts of interest regarding the publication of this article.

\section{References}

[1] S. Benacchio, "Combined state and state derivative control applied to musical string," in Proceedings ICA Conference, Session Paper 4PSA3, Montreal, Canada, 2013.

[2] R. Venugopal and D. S. Bernstein, "State space modeling of an acoustic duct with an end-mounted speaker," Journal of Vibration and Acoustics, vol. 120, no. 3, pp. 770-775, 1998.
[3] Z. Yang and S. Podlech, "Theoretical modeling issue in active noise control for a one-dimensional acoustic duct system," in Proceedings of the 2008 IEEE International Conference on Robotics and Biomimetics, pp. 1249-1254, Banhkok, Thailand, Feburary 2009.

[4] Z. Yang, "Active noise control of a one-dimensional acoustic duct using feedback control techniques: modeling and simulation," W SEAS Transactions, vol. 3, no. 1, pp. 46-54, 2004.

[5] Z. Wu, V. K. Varadan, V. V. Varadan, and K. Y. Lee, "Active absorption of acoustic waves using state-space model and optimal control theory," The Journal of the Acoustical Society of America, vol. 97, no. 2, pp. 1078-1087, 1995.

[6] L. P. R. De Oliveira, P. S. Varoto, P. Sas, and W. Desmet, "A statespace modeling approach for active structural acoustic control," Shock and Vibration, vol. 16, no. 6, pp. 607-621, 2009.

[7] T. Nestorović, J. Lefèvre, and U. Gabbert, "Model-based active control of a piezoelectric structure," Journal of Mechanics, vol. 26, no. 2, 2007.

[8] M. J. Panza, "Closed form solution for acoustic wave equation between two parallel plates using Euler-Maclaurin sum formula," Journal of Sound and Vibration, vol. 277, no. 1-2, pp. 123132, 2004. 
[9] M. J. Panza, "Application of Euler-Maclaurin sum formula to obtain an approximate closed-form Green's function for a twodimensional acoustical space," Journal of Sound and Vibration, vol. 311, no. 1-2, pp. 269-279, 2008.

[10] M. J. Panza, "Mathematical models for finite controllers for a class of bounded acoustic and structural dynamic systems," in Proceedings ACTIVE 04, Williamsburg, VA, USA, 2004.

[11] M. J. Panza, "Euler-Maclaurin closed form finite state space model for a string applied to broadband plate vibrations," Mathematical Problems in Engineering, vol. 2010, Article ID 817194, 14 pages, 2010.

[12] S. I. Hayek, Advanced Mathematical Methods in Science and Engineering, Marcel Dekker, Inc., New York, NY, USA, 2001.

[13] P. Morse, Theoretical Acoustics, McGraw-Hill, New York, NY, USA, 1968.

[14] T. M. Apostal, Calculus, Blaisdell, New York, NY, USA, 1969.

[15] M. J. Panza, "Multi-Source noise reduction calculation for industrial spaces with uncertainties in source size and location," in Proceedings of the NOISE-CON, Cleveland, OH, USA, 2003. 


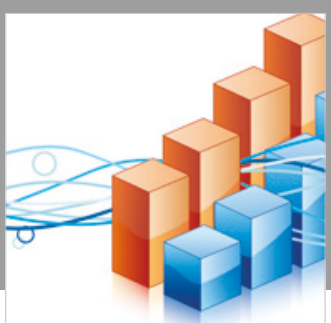

Advances in

Operations Research

\section{-n-m}
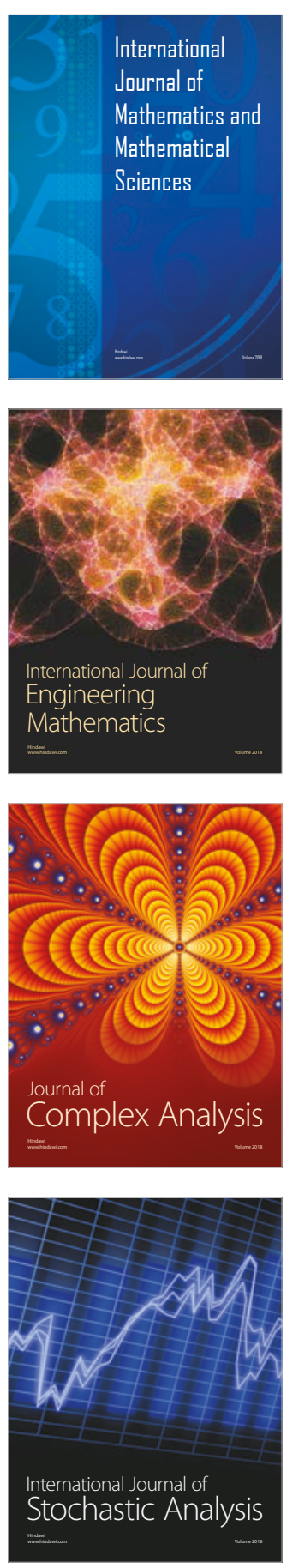
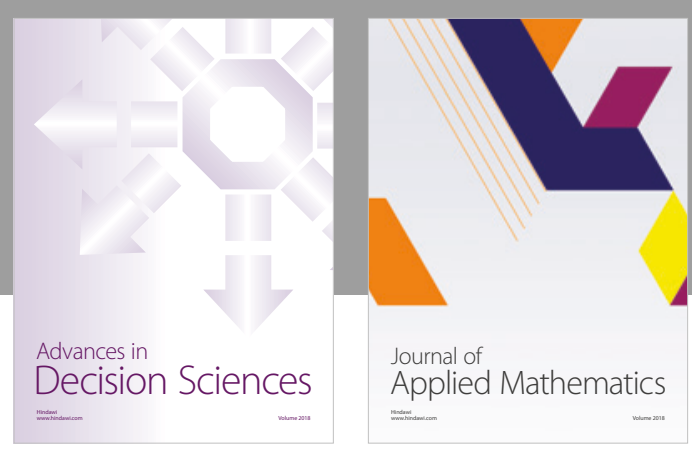

Journal of

Applied Mathematics
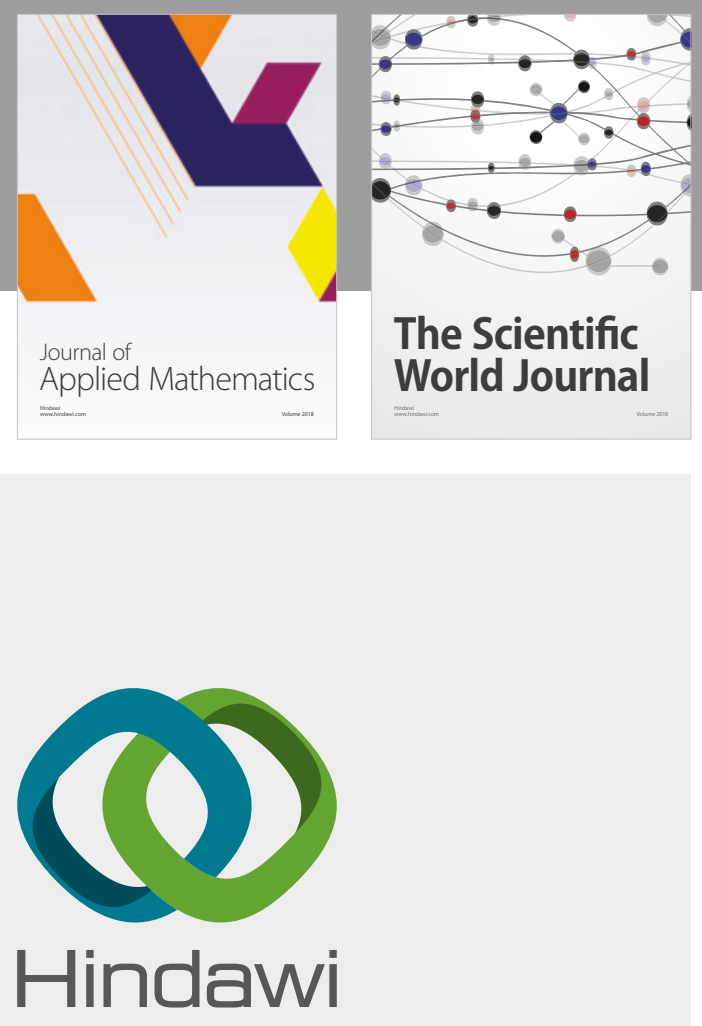

Submit your manuscripts at

www.hindawi.com

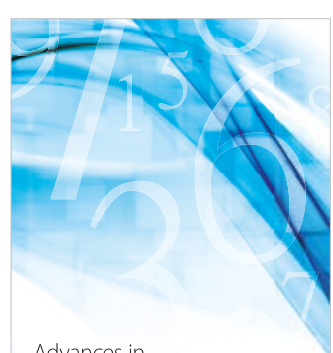

Advances in
Numerical Analysis
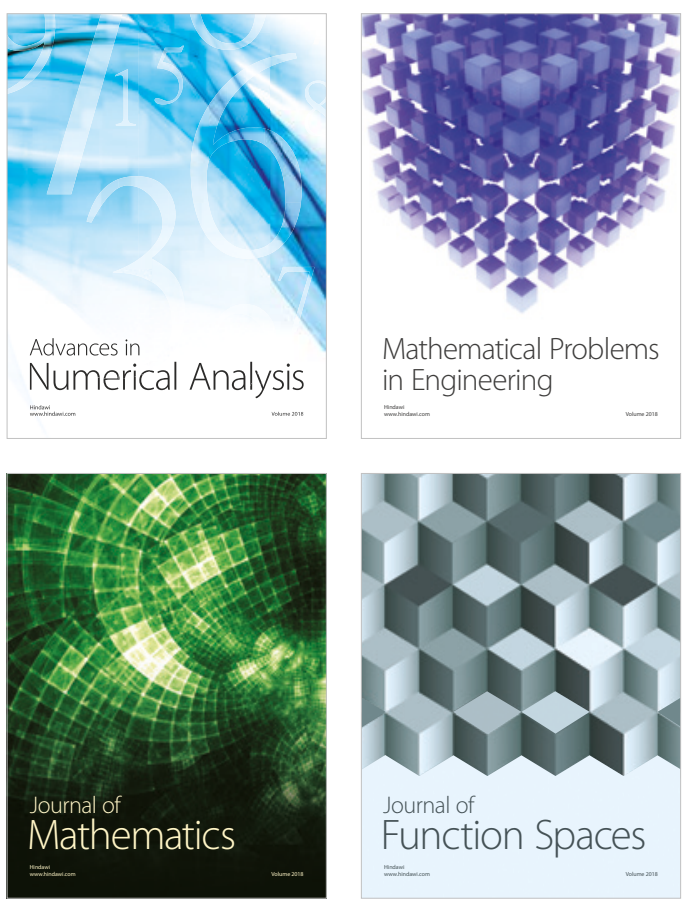

Mathematical Problems in Engineering

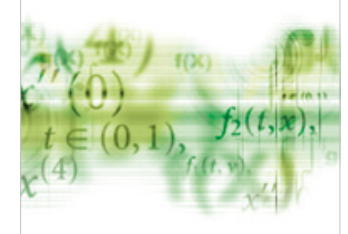

International Journal of

Differential Equations

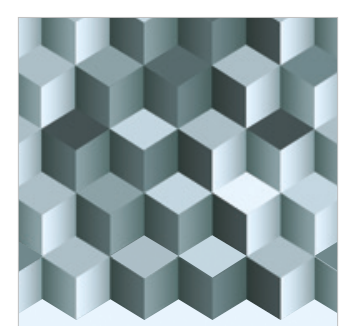

Journal of

Function Spaces

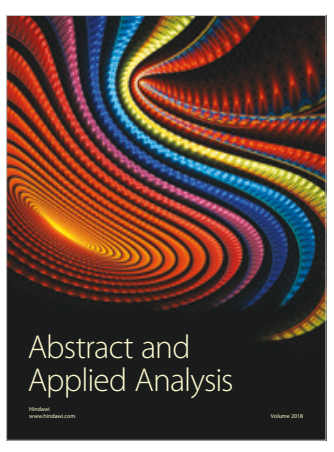

The Scientific

World Journal

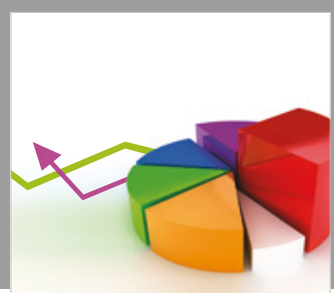

Journal of

Probability and Statistics
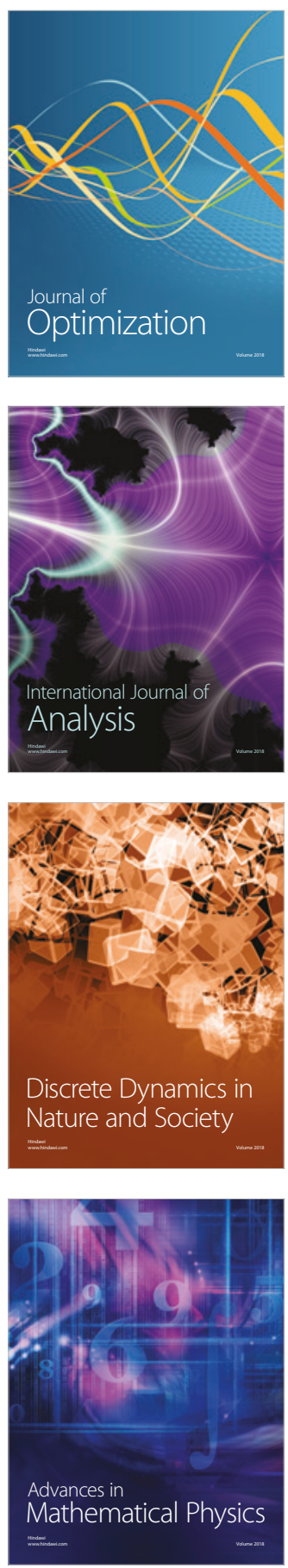